\title{
A VECTOR LATTICE TOPOLOGY AND FUNCTION SPACE REPRESENTATION
}

\author{
BY
}

\author{
W. A. FELDMAN AND J. F. PORTER
}

\begin{abstract}
A locally convex topology is defined for a vector lattice having a weak order unit and a certain partition of the weak order unit, analogous to the order unit topology. For such spaces, called "order partition spaces," an extension of the classical Kakutani theorem is obtained: Each order partition space is lattice isomorphic and homeomorphic to a dense subspace of $C_{c}(X)$ containing the constant functions for some locally compact $X$, and conversely each such $C_{c}(X)$ is an order partition space. $\left(C_{c}(X)\right.$ denotes all continuous real-valued functions on $X$ with the topology of compact convergence.) One consequence is a lattice-theoretic characterization of $C_{c}(X)$ for $X$ locally compact and realcompact. Conditions for an $M$-space to be an order partition space are provided.
\end{abstract}

A classical theorem of Kakutani (see [10]) states that each order unit space with its order unit topology is lattice isometric to a dense subspace of $C(X)$ containing the constant functions with supremum norm for some compact $X$ and, conversely, each such $C(X)$ is an order unit space. (Here $C(X)$ denotes the space of real-valued continuous functions on $X$.) In $\$ 1$ we generalize the concept of an order unit and the topology which it generates and provide an extension of Kakutani's theorem for this setting. Specifically, we consider a vector lattice having a weak order unit $e$ and a partition of $e$ which, in analogy to the order unit case, defines a locally convex topology. Such a space, called an "order partition space," is shown (Theorem 1) to be lattice isomorphic and homeomorphic to a dense subspace of $C_{c}(X)$ containing the constant functions for some locally compact $X$. (Here $C_{c}(X)$ denotes $C(X)$ with the topology of compact convergence.) Conversely, $C_{c}(X)$ for $X$ locally compact is an order partition space. The authors obtained a similar result in Theorem 1 of [5] for the restricted case that $X$ is a countable union of open compact sets. In the remainder of $\S 1$, sufficient conditions are discussed for the order partition topology to be lattice-theoretic-i.e., independent of the choice of $e$

Presented to the Society, January 23, 1976 under the titles Representations of vector lattices having weak order units and Topologies of compact convergence lattice-theoretically; received by the editors March 12, 1976.

AMS (MOS) subject classifications (1970). Primary 46E05, 46A40; Secondary 54C40.

Key words and phrases. Compact convergence, function space, topological $M$-space, vector lattice, weak order unit. 
and the partition of $e$. As one consequence, we obtain a lattice-theoretic characterization of the topology of $C_{c}(X)$ for $X$ locally compact and realcompact. One might wish to compare the "internal" description of $C(X)$ as a vector lattice entailed by our results to other "internal" descriptions of $C(X)$, e.g., those of [1], [2], [7] and [13].

Order partition spaces are (locally convex) $M$-spaces, in the sense of [9]. Conditions under which $M$-spaces can be represented as function spaces with recognizable topologies have been previously studied (e.g., see [10] in the normed case and [9], [11] and [14] in the locally convex case). In \$2 (Theorem 4) we provide necessary and sufficient topological conditions for an $M$-space with a weak order unit to be representable as a dense subspace of $C_{c}(X)$ containing the constant functions for some locally compact $X$ (equivalently, to be an order partition space). These conditions are analyzed in greater detail in Propositions 7 and 8.

1. Representing order partition spaces. Let $V$ be an Archimedean vector lattice. We recall (see [6]) that a weak order unit in $V$ is a positive element $e$ such that $\vee\{v \wedge$ ne: $n=1,2, \ldots\}=v$ for each $v$ in $V$. A collection $\left\{e_{\lambda}\right\}$ of positive elements in $V$ will be called an order partition for $V$ if $V e_{\lambda}$ is a weak order unit in $V$ and if for each $e_{\lambda}$ there is an $e_{\mu}$ so that given $v$ in $V$,

$$
v \wedge n e_{\lambda} \leqslant \delta e_{\mu} \quad(n=1,2, \ldots)
$$

for some real $\delta$ depending on $v$. We will denote $V e_{\lambda}$ by $e$. Corresponding to each $e_{\lambda}$ we can define a seminorm $p_{\lambda}$ by setting, for each $v \in V$,

$$
p_{\lambda}(v)=\inf \left\{\delta>0:|v| \wedge n e_{\lambda} \leqslant \delta e(n=1,2, \ldots)\right\} .
$$

The locally convex topology generated by the collection $\left\{p_{\lambda}\right\}$ will be called an order partition topology for $V$. (As noted in Proposition 2, the same topology is obtained by defining $q_{\lambda}(v)$ to be the infimum of the $\delta$ 's in (*).) By an order partition space we will mean an Archimedean vector lattice together with an order partition topology.

We will show in the proof of Theorem 1 that $C_{c}(X)$ for $X$ locally compact is an order partition space with $V e_{\lambda}=1$. As another example of an order partition space consider the space of bounded functions on the real line $R$ which vanish at infinity. In this space, let $e_{n}$ be the function with value $1 / n$ on $[-n, n]$ and zero elsewhere. Then $\left\{e_{n}\right\}$ is an order partition generating the topology of compact convergence. The space $c_{0}$ of real sequences convergent to zero can be viewed similarly-see the discussion following Corollary 2.

As in [9], we will say a topological vector lattice is an $M$-space if it has a neighborhood base at zero consisting of solid sublattices.

Proposition 1. An order partition space is a Hausdorff M-space. 
Proof. With the above notations, suppose $p_{\lambda}(v)=0$ for each $\lambda$. Then

$$
\underset{\lambda}{\vee}\left(|v| \wedge n e_{\lambda}\right)=0=|\nu| \wedge n\left(\underset{\lambda}{\vee} e_{\lambda}\right)
$$

so that $v \wedge n e=0$ for all $n$. Since $e$ is a weak order unit, $v=0$. One can readily verify the $M$-space condition.

The following lemma will be useful in several arguments of the paper. We denote by $\mathcal{L}$ the set of lattice homomorphisms on $C(X)$ with the weak topology induced by $C(X)$ and by $C_{c}(X)^{\prime}$ the continuous dual of $C_{c}(X)$.

LEMMA 1. (a) Let $X$ be a realcompact space and $f$ a nonnegative function in $C(X)$. Then the subspace $\{x \in X: f(x)>0\}$ is homeomorphic to the subspace $(\phi \in \mathcal{L}: \phi(f)=1\}$. In particular, if $f$ is never zero, $X$ is homeomorphic to $\{\phi \in \mathcal{L}: \phi(f)=1\}$.

(b) Let $X$ be a completely regular space and $f$ a nonnegative function in $C(X)$. Then the subspace $\{x \in X: f(x)>0\}$ is homeomorphic to the subspace $\{\phi$ $\left.\in \mathfrak{L} \cap C_{c}(X)^{\prime}: \phi(f)=1\right\}$. In particular, if $f$ is never zero, $X$ is homeomorphic to $\left\{\phi \in \mathfrak{L} \cap C_{c}(X)^{\prime}: \phi(f)=1\right\}$.

Proof. (b) For $X$ completely regular, the continuous dual of $C_{c}(X)$ consists of the regular finite Borel measures on $X$ having compact support. It follows readily that the continuous members of $\mathfrak{L}$ are the point measures on $X$. Associating to each continuous $\phi \in \mathcal{L}$ with $\phi(f)=1$ the point support of $\phi$ establishes a one-to-one correspondence between the sets in question. For the topologies, suppose net $\phi_{\alpha} \rightarrow \phi$ for $\phi_{\alpha}$ and $\phi$ in $\{\theta \in \mathcal{L}: \theta(f)=1$ and $\theta$ is continuous\} with support points $x_{\alpha}$ and $x$. Writing $\phi_{\alpha}=\beta_{\alpha} x_{\alpha}^{\prime}$ and $\phi=\beta x^{\prime}$, where $x_{\alpha}^{\prime}$ and $x^{\prime}$ are the unit point measures at $x_{\alpha}$ and $x$, we obtain that $\beta_{\alpha} \rightarrow \beta$ since $\phi_{\alpha}(1) \rightarrow \phi(1)$. Since $\{f \wedge n 1\}$ converges to $f$, then $\phi(1) \neq 0$, so that $g\left(x_{\alpha}\right) \rightarrow g(x)$ for each $g$ in $C(X)$. Thus $x_{\alpha} \rightarrow x$ in $X$. Continuity in the other direction follows similarly.

(a) For $X$ realcompact, the order dual of $C(X)$ coincides with the continuous dual of $C_{c}(X)$ (see [8, p. 179]). Thus the proof of (b) applies.

Corollary 1. (a) For $X$ and $Y$ realcompact, $C(X)$ and $C(Y)$ are lattice isomorphic if and only if $X$ and $Y$ are homeomorphic.

(b) For $X$ and $Y$ completely regular, $C_{c}(X)$ and $C_{c}(Y)$ are lattice isomorphic and homeomorphic if and only if $X$ and $Y$ are homeomorphic.

Proof. (b) Let $T: C_{c}(X) \rightarrow C_{c}(Y)$ be a lattice isomorphism and homeomorphism. For $y$ in $Y, y^{\prime} \circ T$ is a point measure on $X$, say $\alpha x^{\prime}$ for $\alpha>0$. (Here the notations and arguments are as in the previous proof.) If $f$ is positive and never zero in $C_{c}(X)$ then $(T f)(y)=\alpha f(x)>0$ so that $T f$ is positive and 
never zero in $C_{c}(Y)$. It now follows from (b) of Lemma 1 that $X$ and $Y$ are homeomorphic.

(a) This follows similarly, using (a) of Lemma 1.

Given an $M$-space $V$ with weak order unit $e$ we denote by $L(V)$ the set of all continuous lattice homomorphisms $x$ on $V$ having $x(e)=1$, together with the weak topology induced by $V$. We will refer to $L(V)$ as the carrier space of $V$ for $\left\{e_{\lambda}\right\}$.

LEMMA 2. Let $V$ be a Hausdorff $M$-space with weak order unit e. Then $V$ is lattice isomorphic to a subspace of the space $C(L(V))$ of all continuous realvalued functions on $L(V)$ and $V$ is dense in $C(L(V))$ with respect to the topology of compact convergence. The image of $e$ is 1 .

Proof. We will first show that $L(V)$ separates the points of $V$. It is known (Corollary 1 of [9]) that the set $\mathcal{L}(V)$ of all continuous lattice homomorphisms on $V$ separates the points of $V$. Let $T$ denote those members $y$ of $L(V)$ having $y(e) \neq 0$. Assuming that $T$ does not separate the points of $V$, there is a $v \neq 0$ in $V$ such that $y(v)=0$ for all $y$ in $T$. Then for all $y$ in $L(V)$ either $y(e)=0$ or $y(v)=0$, implying that $y(v \wedge n e)=y(v) \wedge n y(e)=0$. Since $f(V)$ separates the points of $V$, we have $\vee\{v \wedge n e: n=1,2, \ldots\}=0$, contradicting the fact that $e$ is a weak order unit. Since each member of $T$ is a scalar multiple of an element in $L(V)$ we obtain that $L(V)$ separates the points of $V$.

The mapping $\Phi$ from $V$ into $C(L(V))$ defined by $[\Phi v](x)=x(v)$ for each $x$ in $L(V)$ is a lattice homomorphism having $\Phi(e)=1$. Since $L(V)$ separates the points of $V$ the mapping $\Phi$ is one-to-one, and we will not distinguish $V$ from its image in $C(L(V))$. By the Stone-Weierstrass Theorem, $V$ is dense in $C(L(V))$ with the topology of compact convergence.

THEOREM 1. A complete topological vector lattice $(V, \tau)$ is homeomorphic and lattice isomorphic to $C_{c}(X)$ for some locally compact space $X$ if and only if $V$ has an order partition whose order partition topology is $\tau$. Every order partition space is homeomorphic and lattice isomorphic to a dense sublattice of $C_{c}(X)$ containing the constant functions for some locally compact $X$. In each case $X$ is unique up to homeomorphism.

Proof. The uniqueness of $X$ is a consequence of Corollary 1. Let $V$ be an order partition space. We choose $X$ to be the space $L(V)$ and view $V$ as the dense subspace of $C_{c}(X)$ discussed in the preceding lemma. We will first show that for each seminorm $p_{\lambda}$ of the order partition topology there is a compact subset $F_{\lambda}$ of $X$ such that $p_{\lambda}$ is the supremum seminorm on $F_{\lambda}$. We let $E_{\lambda}$ denote the set $\left\{x \in X: e_{\lambda}(x)>0\right\}$. Since $|v(x)| \wedge n e_{\lambda}(x)$ is zero if $x \notin E_{\lambda}$ and is less than or equal to $|v(x)|$ if $x \in E_{\lambda}$, we have 


$$
p_{\lambda}(v) \leqslant \sup \left\{|v(x)|: x \in E_{\lambda}\right\} .
$$

(Recall that $e(x)=1$.) On the other hand, for $x \in E_{\lambda}$ and $n$ sufficiently large,

$$
|v(x)| \wedge n e_{\lambda}(x)=|v(x)| .
$$

From the definition of $p_{\lambda}$ we have $|v| \wedge n e_{\lambda} \leqslant p_{\lambda}(v) e$. Thus

$$
p_{\lambda}(v)=\sup \left\{|v(x)|: x \in E_{\lambda}\right\} .
$$

We show that the set $F_{\lambda}=\bar{E}_{\lambda}$ is compact. In the algebraic dual of $V$ with the weak topology induced by $V$ it is clear that $X$ is closed and that the intersection of $X$ and the polar $U_{\lambda}^{\circ}$ of the set $U_{\lambda}=\left\{v \in V: p_{\lambda}(v) \leqslant 1\right\}$ is compact. This intersection is $\left\{x \in X:|v(x)| \leqslant p_{\lambda}(v)\right\}$, which by $(\dagger)$ contains $E_{\lambda}$.

In fact, $E_{\lambda}=U_{\lambda}^{\circ} \cap X$, which we will utilize below. To verify this, we let $x$ be in $X \backslash \bar{E}_{\lambda}$. There is a function $f$ in $C(X)$ with $f(x)=2$ and $f\left(\bar{E}_{\lambda}\right)=0$. Since $V$ is dense in $C_{c}(X)$ there is a point $v \in V$ such that $v\left(\bar{E}_{\lambda}\right) \subseteq\left[-\frac{1}{2}, \frac{1}{2}\right]$ and $v(x)>\frac{3}{2}$. It follows that $v$ is in $U_{\lambda}$ and, hence, $x$ is not in $U_{\lambda}^{\circ}$.

Given $x$ in $X$, there is an $\varepsilon>0$ and a finite union $K$ of sets $\bar{E}_{\lambda}$ such that $\sup \{|v(y)|: y \in K\} \leqslant \varepsilon$ implies $|v(x)| \leqslant 1$, since $x$ is continuous. Arguing as above, one concludes that $x$ is in the compact set $K$. Thus $X=\cup \bar{E}_{\lambda}$. (We will see in the next paragraph that $X=\cup E_{\lambda}$.)

By definition, for each $e_{\mu}$ there is an $e_{\lambda}$ and $\delta>0$ such that $e \wedge n e_{\mu} \leqslant \delta e_{\lambda}$. For $x$ in $X$ this becomes $1 \wedge n e_{\mu}(x) \leqslant \delta e_{\lambda}(x)$; it follows that $e_{\lambda}$ is greater than or equal to $1 / \delta$ on $E_{\mu}$. By the continuity of $e_{\lambda}$ we conclude $\bar{E}_{\mu} \subseteq E_{\lambda}$. It is immediate that $X$ is locally compact. Also each compact subset of $X$ is contained in a finite union of sets of the form $E_{\lambda}$, so that the order partition topology is equivalent to the topology of compact convergence. If $V$ is complete, then, of course, $V$ is all of $C_{c}(X)$.

Finally, given $C_{c}(X)$ for some locally compact space $X$, for each $x$ in $X$ we choose a function $f_{x}$ in $C(X)$ with $0 \leqslant f_{x} \leqslant 1$ such that $f_{x}$ is one on a neighborhood $W_{x}$ of $x$ and the closure of $\left\{y \in X: f_{x}(y)>0\right\}$ is compact. We let the collection $\left\{e_{\lambda}\right\}$ consist of all suprema of finite collections of the functions $f_{x}$. Clearly $\vee e_{\lambda}$ is one and each $e_{\lambda}$ has compact support. Given $e_{\lambda}$ with support $\bar{E}_{\lambda}$, let $\left\{W_{x_{i}}: i=1, \ldots, n\right\}$ be a cover for $\bar{E}_{\lambda}$ and let $e_{\mu}$ $=\bigvee\left\{f_{x_{i}}: i=1, \ldots, n\right\}$. For $g$ in $C(X)$, by setting $\delta=\sup \left\{|g(x)|: x \in \bar{E}_{x}\right\}$, we obtain for all $n$ that $|g| \wedge n e_{\lambda} \leqslant \delta e_{\mu}$. In fact, as argued above, $\delta=p_{\lambda}(g)$. Since each compact subset of $X$ is contained in the support of some $e_{\lambda}$. We conclude that $C_{c}(X)$ is an order partition space.

COROLlaRY 2. An order partition space is metrizable if and only if its carrier space is $\sigma$-compact. 
Proof. If the order partition space is metrizable then $X=\cup \bar{E}_{\lambda}$ for a countable collection $\left\{e_{\lambda}\right\}$. Conversely, if $X$ is $\sigma$-compact then $C_{c}(X)$ is metrizable.

Given an order partition space $V$, it will follow from Propositions 5 and 6 of $\S 2$ that for each $v$ in $V,\{v \wedge n e\}$ converges topologically to $v$. Furthermore, if $\phi$ is a continuous nonzero lattice homomorphism on $V$ then $\phi(e) \neq 0$, so that $\phi / \phi(e)$ is in the carrier space of $V$.

To illustrate the representation of Theorem 1 , we observe that $c_{0}$, the space of real sequences convergent to zero, with the topology of pointwise convergence, is an order partition space. Let $e_{n}$ be the sequence with entries $1 / k$ for $k \leqslant n$ and 0 for $k>n$. Then $\left\{e_{n}\right\}$ is an order partition for $c_{0}$ for which the corresponding order partition topology is the topology of pointwise convergence. By Theorem 1 this space can be represented as a dense sublattice of $C_{c}(X)$ containing the constant functions. It is easy to verify that $X$ is homeomorphic to the set $N$ of natural numbers and the image of the sequence $\{1 / j\}$ (i.e., $\vee e_{n}$ ) is the constant function 1 . In contrast, as a locally convex $M$ space (disregarding the additional order partition structure) $c_{0}$ with the topology of pointwise convergence is representable as a subspace of some $C(X)$ by a theorem of Jameson (see [9]); however, this representation does not contain the constant functions and $X$ is larger than $N$. A similar discussion can be given for the bounded functions vanishing at infinity.

Proposition 2. Given an order partition space $V$ the seminorm

$$
p_{\lambda}(v)=\inf \left\{\beta>0:|v| \wedge n e_{\lambda} \leqslant \beta e(n=1,2, \ldots)\right\}
$$

is equivalent to the seminorm

$$
q_{\lambda}(v)=\inf \left\{\beta>0:|v| \wedge n e_{\lambda} \leqslant \beta e_{\mu}(n=1,2, \ldots)\right\}
$$

where $e_{\mu}$ satisfies (*) of the definition of an order partition.

Proof. Since $e_{\mu} \leqslant e$ it is clear that $q_{\lambda}(v) \geqslant p_{\lambda}(v)$. On the other hand, using the representation of Theorem 1 we showed that $E_{\lambda} \subseteq E_{\mu}$, where $E_{\rho}$ $=\left\{x \in X: e_{\rho}(x)>0\right\}$. Since $e_{\mu}(x) \geqslant \delta_{1}>0$ for $x$ in $E_{\lambda}$ and $e_{\lambda}(x)=0$ for $x$ not in $E_{\lambda}$, we obtain $p_{\lambda}(v) \geqslant\left(1 / \delta_{1}\right) q_{\lambda}(v)$.

An order partition which gives rise to a complete topology and a realcompact carrier space will be called a proper order partition.

THEOREM 2. All proper order partitions for a vector lattice $V$ generate the same order partition topology. A proper order partition space $V$ can be identified with $C_{c}(X)$ for some realcompact and locally compact $X$. Any order partition $\left\{e_{\lambda}\right\}$ in $V$ generates the (coarser) topology induced from $C_{c}(Y)$ for a subspace $Y$ of $X$. 
Proof. The first statements follow from Corollary 1 and Theorem 1. Given $\left\{e_{\lambda}\right\}$, its carrier space can be embedded in $\{x \in X: e(x)>0\}$ by Lemma 1. The result is again a consequence of Theorem 1 .

Proposirion 3. For $X$ locally compact, any complete order partition topology for $C(X)$ is coarser than that of $C_{c}(\nu X)$, where $X$ is regarded as a subspace of its Hewitt realcompactification $\nu X$.

Proof. Analogously to the proof of Theorem 2, the carrier space must be homeomorphic to a subspace of $\nu X$.

We provide examples which show that neither completeness nor realcompactness can be eliminated from Theorem 2 .

We consider two order partitions for the space $C_{b}(R)$ of all bounded continuous real-valued functions on the real line $R$ :

(1) the collection $\left\{f_{n}\right\}$ of functions chosen so that $0<f_{n}<1, f_{n}$ is one on $[-n, n]$ and $f_{n}$ is zero outside $[-n-1, n+1]$;

(2) the function 1 on $R$.

The order partition topology for (1) is the topology of compact convergence which is not complete, whereas the order partition topology for (2) is the supremum norm topology which is complete. However, the carrier spaces for (1) and (2) are the same as for $C_{c}(R)$ and $C_{c}(\beta R)$, respectively, which are realcompact by Lemma 1. (Here $\beta R$ denotes the Stone-Cech compactification of $R$.)

To show the necessity of realcompactness, we let $X$ be a locally compact and pseudocompact (but not compact) space, e.g., see p. 231 of [3]. The order partition $\{1\}$ for $C(X)$ and any order partition giving the topology of compact convergence (e.g., as in Theorem 1) generate complete but distinct order partition topologies. The space $X$ cannot be realcompact.

THEOREM 3. For a complete order partition space $V$ with order partition $\left\{e_{\lambda}\right\}$, each of the following conditions implies that $\left\{e_{\lambda}\right\}$ is a proper order partition:

a. The supremum of $\left\{e_{\lambda}\right\}$ in the order bidual of $V$ coincides with its supremum in $V$, e.g., if $V$ is minimal (see $[12, p .157])$.

b. Every positive linear functional on $V$ is continuous.

c. The collection $\left\{e_{\lambda}\right\}$ is countable.

Proof. We will show that each of the conditions cited implies that the carrier space is realcompact. That this is so for condition (c) is evident from the fact that $X=\cup E_{\lambda}$ where $E_{\lambda}=\left\{x \in X: e_{\lambda}(x)>0\right\}$, as noted in the proof of Theorem 1, implying that $X$ is $\sigma$-compact.

For (a) and (b) we let $X$ denote the carrier space for $V$ and identify $V$ with $C_{c}(X)$ (and $e$ with 1 ) as in Theorem 1. For $\nu X$, the Hewitt realcompactification of $X, C(X)$ is lattice isomorphic to $C(\nu X)$ so that by Lemma $1, \nu X$ 
$=\{\phi \in \mathcal{L}: \phi(1)=1\}$. Given (b) this latter set is $X$; hence $X=\nu X$ and is realcompact. Given (a), we assume without loss of generality that $\left\{e_{\lambda}\right\}$ is directed upward. It then follows for any $x$ in $\nu X$ that in the order bidual of $V$,

$$
\vee\left(e_{\lambda}(x)\right)=\left(\vee e_{\lambda}\right)(x)=e(x)=1,
$$

so that $e_{\mu}(x)>0$ for some $e_{\mu}$. But then $x\left(v \wedge n e_{\mu}\right)=x(v)$ for $n$ sufficiently large, so that the image under $x$ of $\left\{v \in V:|v| \wedge n e_{\mu} \leqslant e\right\}$ is contained in $[-1,1]$. Since this set is $\left\{v \in V: p_{\mu}(v) \leqslant 1\right\}, x$ must be continuous. Thus $\nu X=X$ so that $X$ is realcompact.

The techniques of.Theorem 3 allow us to state the following supplement to Theorem 2.

Proposition 4. Let $X$ be realcompact and let $\left\{e_{\lambda}\right\}$ be an order partition for $C(X)$ such that the suprema of $\left\{e_{\lambda}\right\}$ and $\{1 \wedge$ ne $\}$ in the order bidual of $C(X)$ coincide with their respective suprema in $C(X)$. Then the corresponding order partition topology is the topology of compact convergence and $X$ is locally compact.

Proof. By Theorem 1 the order partition space corresponding to $\left\{e_{\lambda}\right\}$ is a dense subspace of $C_{c}(Y)$ where $Y$ is the locally compact space consisting of all lattice homomorphisms $\phi$ on $C(X)$ having $\phi(e)=1$ and which are continuous with respect to the order partition topology. The bidual condition $\vee(1 \wedge n e)=1$ implies that $e$ is never zero in $X$, so, by Lemma $1, X$ is homeomorphic to $\{\phi \in \mathcal{L}: \phi(e)=1\}$. For $\phi$ in this latter set the bidual condition $\vee e_{\lambda}=e$ implies that $\phi\left(e_{\mu}\right)>0$ for some $e_{\mu}$. Given $f$ in $C(X)$ with $p_{\mu}(f) \leqslant 1$ we obtain (since $|f| \wedge n e_{\mu} \leqslant e$ ) that $|\phi(f)| \wedge n \phi\left(e_{\mu}\right) \leqslant 1$. Hence $|\phi(f)| \leqslant 1$, which implies that $\phi$ is continuous. Thus $X$ is homeomorphic to $Y$.

We remark that condition (*) in the definition of an order partition is similar to the stronger condition $v \wedge n e_{\lambda} \leqslant \beta e_{\lambda}(n=1,2, \ldots)$. Such elements $e_{\lambda}$ (called semiorder-units) were studied in [4] and [5]. In the presence of the weak order unit $V e_{\lambda}$ this stronger condition implies that the set $E_{\lambda}=\left\{x \in X: e_{\lambda}(x)>0\right\}$ is both open and compact (see the proof of Theorem 1). Theorem 1 is valid under this additional hypothesis if the condition " $X$ is a union of open compact subsets" is substituted for local compactness. (Compare Theorem 1 in [5].)

The condition $v \wedge n e_{\lambda} \leqslant \beta e(n=1,2, \ldots)$ is not equivalent to (*). Defining $e_{k}(k=1,2, \ldots)$ in $C[0,1]$ by $e_{k}(x)=k x$ for $x \leqslant 1 / k$ and $e_{k}(x)=1$ for $x>1 / k$ one obtains a system $\left\{e_{k}\right\}$ satisfying the weaker condition but not (*), with $e=V e_{k}=1$. We note that by defining $e_{k}^{\prime}$ to be 0 for $x<(k+1)^{-1}$ and 1 for $x>1 / k$, together with the connecting line segment, one obtains an order partition for $C[0,1]$ having carrier space $(0,1]$. 
2. Order partition spaces as $M$-spaces. Let $V$ be an $M$-space whose topology is generated by the collection of $M$-seminorms $\left\{p_{\lambda}\right\}$. (By an $M$-seminorm we mean a seminorm $p_{\lambda}$ such that $p_{\lambda}(v) \leqslant p_{\lambda}(w)$ if $0 \leqslant v \leqslant w$ and $p_{\lambda}(v \vee w)$ $=p_{\lambda}(v) \vee p_{\lambda}(w)$ for $v \geqslant 0$ and $w \geqslant 0$.) We will say that a positive element $e$ in $V$ satisfies the quotient condition if for each $p_{\lambda}$, the image of $e$ in the quotient of $V$ by the ideal $\left\{v \in V: p_{\lambda}(v)=0\right\}$ is an order unit and the order unit topology coincides with the topology induced by $p_{\lambda}$. We will say that $e$ satisfies the orthogonality condition if for each $p_{\lambda}$ there exist a positive element $v_{\lambda}$ in $V$ and an $M$-seminorm $p_{\mu} \geqslant p_{\lambda}$ such that $p_{\lambda}\left(e-v_{\lambda}\right)=0$ and $v_{\lambda} \wedge|w|=0$ whenever $p_{\mu}(w)=0$.

Theorem 4, the main result of this section, shows that an order partition space is equivalent to a Hausdorff $M$-space with these conditions.

Proposition 5. Given an order partition space, the weak order unit corresponding to the order partition satisfies the quotient condition and the orthogonality condition.

Proof. Let $V$ be an order partition space. By Theorem $1, V$ can be regarded as a dense subspace of $C_{c}(X)$ with weak order unit 1 . Since each $p_{\lambda}$ is the supremum seminorm on a compact subset $\bar{E}_{\lambda}$ of $X$, the quotient of $V$ by the ideal $\left\{v \in V: p_{\lambda}(v)=0\right\}$ is an order unit subspace of $C\left(\bar{E}_{\lambda}\right)$. To show the orthogonality condition, given $e_{\lambda}$ we set $v=e \wedge \delta e_{\mu}$, where $e \wedge n e_{\lambda} \leqslant \delta e_{\mu}$ for all $n$. Then $v$ is 1 on $\bar{E}_{\lambda}$ and 0 on $X \backslash E_{\mu}$, so that the condition is evident.

Proposition 6. Let e satisfy the quotient condition in a Hausdorff $M$-space $V$. Then $e$ is a weak order unit in $V$ and for each $v$ in $V$ the sequence $\{v \wedge n e\}_{n=1}^{\infty}$ converges to $v$ topologically. Furthermore, each nontrivial continuous lattice homomorphism on $V$ is nonzero at $e$.

Proof. Given $v$ in $V$ and seminorm $p_{\lambda}$, since $e$ satisfies the quotient condition, there exist $\mu_{\lambda}>0$ in $V$ and real $\beta>0$ such that $p_{\lambda}\left(u_{\lambda}\right)=0$ and $v-u_{\lambda} \leqslant \beta e$. Hence for all $n$,

$$
p_{\lambda}[v-v \wedge n e] \leqslant p_{\lambda}\left[v-\left(v-u_{\lambda}\right) \wedge n e\right] \leqslant p_{\lambda}\left[v-u_{\lambda}-\left(v-u_{\lambda}\right) \wedge n e\right] .
$$

For $n>\beta$ this last expression is zero. Thus $v \wedge n e$ converges to $v$ topologically. It now follows that $v=\vee(v \wedge n e)$, since otherwise $v \wedge n e \leqslant w<v$ for some $w$ in $V$ and all $n$, implying that $p_{\mu}(v-v \wedge n e) \geqslant p_{\mu}(v-w)>0$ for some seminorm $p_{\mu}$. For the last statement of the theorem we note that for a continuous lattice homomorphism $\phi$, if $\phi(e)=0$ then $\phi(v \wedge n e)=0$ for all $n$, which implies by the topological convergence that $\phi(v)=0$.

THEOREM 4. Let $V$ be a topological vector lattice. Then $V$ is an order partition space if and only if it is a Hausdorff $M$-space with a positive element e satisfying 
the quotient condition and the orthogonality condition.

Proof. The sufficiency has been shown in Propositions 1 and 5. For the necessity we first note by Proposition 6, $e$ is a weak order unit. For seminorm $p_{\lambda}$, it is clear that the positive element $v_{\lambda}$ of the orthogonality condition can be replaced by the element $e_{\lambda}=e \wedge v_{\lambda}$. Since $e_{\lambda} \leqslant t<e$ for all $\lambda$ implies $p_{\lambda}(t-e) \leqslant p_{\lambda}\left(e-e_{\lambda}\right)=0$ for all $\lambda$, we conclude that $e=V e_{\lambda}$. We will next show that the condition (*) of an order partition is satisfied. Given $e_{\lambda}$, the orthogonality condition states that there is a seminorm $p_{\mu}$ such that $|w| \wedge e_{\lambda}$ $=0$ whenever $p_{\mu}(w)=0$. Since $e_{\mu}$ is an order unit in the quotient of $V$ by $\left\{v \in V: p_{\mu}(v)=0\right\}$, for each $v$ in $V$ we have $v \leqslant \beta e_{\mu}+r_{\mu}$ for some $\beta>0$ and positive $r_{\mu}$ with $p_{\mu}\left(r_{\mu}\right)=0$. It follows that $v \wedge n e_{\lambda} \leqslant \beta e_{\mu} \wedge n e_{\lambda}+r_{\mu} \wedge n e_{\lambda}$ $=\beta e_{\mu} \wedge n e_{\lambda} \leqslant \beta e_{\mu}$, since $r_{\mu} \wedge e_{\lambda}=0$ by the orthogonality condition.

Finally, we will show that the order partition topology agrees with the $M$ space topology of the seminorms $\left\{p_{\lambda}\right\}$. By the quotient condition we can assume that

$$
p_{\mu}(v)=\inf \left\{\beta>0:|v| \leqslant \beta e_{\mu}+r_{\mu} \text { and } p_{\mu}\left(r_{\mu}\right)=0\right\} \text {. }
$$

The argument of the preceding paragraph enables us to conclude that $q_{\lambda}(v) \leqslant p_{\mu}(v)$, where $q_{\lambda}$ is the order partition seminorm corresponding to $e_{\lambda}$. On the other hand, if $|v| \wedge n e_{\lambda} \leqslant \beta e$ then, since $e_{\lambda}$ and $e$ have the same image in the quotient of $V$ by $\left\{w \in V: p_{\lambda}(w)=0\right\}$, we obtain $|v| \leqslant \beta e_{\lambda}+s_{\lambda}$ for some $s_{\lambda}$ with $p_{\lambda}\left(s_{\lambda}\right)=0$. Thus $p_{\lambda}(v) \leqslant q_{\lambda}(v)$.

Proposition 7. Let $V$ be a Hausdorff $M$-space having a positive element $e$ which satisfies the quotient condition and the orthogonality condition. If $d$ is an element of $V$ such that $\phi(d)>0$ for all nonzero continuous lattice homomorphisms $\phi$ on $V$ then $d$ also satisfies the quotient condition and the orthogonality condition.

Proof. By Theorem 4, $V$ is an order partition space and can be represented (see Theorem 1) as a dense subspace of $C_{c}(X)$. Thus the quotient of $V$ by $\left\{v \in V: p_{\lambda}(v)=0\right\}$ can be viewed as an order unit subspace of $C(K)$ for a compact subset $K$ of $X$ and since $d$ is strictly positive in $C(X)$ its image in $C(K)$ is an order unit. To prove the orthogonality condition we let $e_{\mu}$ be a positive member of $V$ with compact support $T$ in $X$ whose image in $C(K)$ is an order unit. (This is possible since $V$ is an order partition space.) Letting $v_{\lambda}=d \wedge \beta e_{\mu}$, where $d(x) \leqslant \beta e_{\mu}(x)$ for $x$ in $K$, we have $p_{\lambda}\left(d-v_{\lambda}\right)=0$ and if $\sup \{|w(x)|: x \in T\}=0$ then $|w| \wedge v_{\lambda}=0$.

It is a consequence of Proposition 7 and the proof of Theorem 4 that given an order partition space $V$, if $d$ is an element of $V$ such that $\phi(d)>0$ for all nonzero continuous lattice homomorphisms $\phi$ on $V$ then there is an order 
partition corresponding to $d$ generating the topology of $V$.

Proposition 8. Let $V$ be a Hausdorff $M$-space generated by the $M$-seminorms $\left\{p_{\lambda}\right\}$ and let $e$ be a positive element in $V$. Then e satisfies the quotient condition if and only if $\{v \wedge n e\}$ converges to $v$ (topologically) for every $v$ in $V$ and for each $p_{\lambda}$, all $M$-seminorms on $V$ with value 1 on $e$ and dominated by some multiple of $p_{\lambda}$ are dominated by a fixed multiple of $p_{\lambda}$.

Proof. Let $e$ satisfy the quotient condition. By Proposition 6, $\{v \wedge n e\}$ converges to $v$ for each $v$ in $V$. By the quotient condition, there is an $\alpha>0$ such that, given $p_{\lambda}(v)<1,|v|<\alpha e+t$ for some $t$ in $V$ with $p_{\lambda}(t)=0$. Thus if $p$ is an $M$-seminorm with $p(e)=1$ dominated by a multiple of $p_{\lambda}$, it follows that $p(t)=0$ so that $p(v) \leqslant \alpha p(e)+p(t)=\alpha$. This shows $p<\alpha p_{\lambda}$. Suppose the hypotheses of the converse are satisfied. Let $y$ be any continuous nonzero lattice homomorphism on the quotient of $V$ by $\left\{v \in V: p_{\lambda}(v)=0\right\}$ in the norm topology induced by $p_{\lambda}$. Letting $\left(^{-}\right)$denote images in this quotient, we note that $\{\bar{v} \wedge n \bar{e}\}$ converges to $\bar{v}$ for each $v$ in $V$ and thus $y(\bar{e})>0$. Since $y$ is continuous, the homomorphism $y / y(\bar{e})$ is dominated by a multiple of the norm induced by $p_{\lambda}$. Thus there is a fixed $\beta>0$ such that $(y / y(\bar{e}))(\bar{v})<$ $\beta p_{\lambda}(\bar{v})$ for each continuous nonzero lattice homomorphism $y$; i.e., $y(v)<$ $y\left(\left[\beta p_{\lambda}(\bar{v})\right] \bar{e}\right)$. Arguing as in the proof of Proposition 6, we conclude that $\bar{e}$ is a weak order unit in the quotient, so that, by Lemma $2, \bar{v}<\left[\beta p_{\lambda}(\bar{v})\right] \bar{e}$. It follows that $\bar{e}$ is an order unit whose norm $q$ is less than or equal to $\beta p_{\lambda}$. The equivalence of $q$ and $p_{\lambda}$ is immediate, since $p_{\lambda}$ is monotone and $\bar{v}<q(\bar{v}) \bar{e}$.

\section{REFERENCES}

1. F. W. Anderson, Approximation in systems of real-valued functions, Trans. Amer. Math. Soc. 103 (1962), 249-271. MR 25 \#436.

2. E. Binz, Notes on a characterization of function algebras, Math. Ann. 186 (1970), 314-326. MR 41 \#8992.

3. J. Dugundji, Topology, Allyn and Bacon, Boston, Mass., 1966. MR 33 \# 1824.

4. W. A. Feldman and J. F. Porter, Compact convergence and the order bidual for $C(X)$, Pacific J. Math. 57 (1975), 113-124. MR 51 \#11074.

5. - Order units and base norms generalized for convex spaces, Proc. London Math. Soc. (3) 33 (1976), 299-312.

6. D. H. Fremlin, Topological Riesz spaces and measure theory, Cambridge Univ. Press, London, 1974.

7. M. Henriksen and D. G. Johnson, On the structure of a class of archimedean lattice-ordered algebras, Fund. Math. 50 (1961), 73-94. MR 24 \# A3524.

8. E. Hewitt, Linear functionals on spaces of continuous functions, Fund. Math. 37 (1950), 161-189. MR 13, 147.

9. G. J. O. Jameson, Topological M-spaces, Math. Z. 103 (1968), 139-150. MR 36 \#5655.

10. S. Kakutani, Concrete representation of abstract (M)-spaces, Ann. of Math. (2) 42 (1941), 994-1028. MR 3, 205.

11. R. G. Kuller, Locally convex topological vector lattices and their representations, Michigan Math. J. 5 (1958), 83-90. MR 20 \#4173. 
12. A. L. Peressini, Ordered topological vector spaces, Harper and Row, New York, 1967. MR 37 \#3315.

13. D. Plank, Closed l-ideals in a class of lattice-ordered algebras, Illinois J. Math. 15 (1971), 515-524. MR 43 \#6143.

14. C. Portenier, Espaces de Riesz, espaces de fonctions et espaces de sections, Comment. Math. Helv. 46 (1971), 289-313. MR 45 \#855.

Dipartmient of Mathematics, University of Arransas, FayetTEVILLE, Arkansas 72701 